\title{
475394 - ULTRASOUND ASSISTED SPINAL ANESTHESIA IN A PATIENT WITH SCOLIOSIS AND PREVIOUS BACK SURGERY
}

\author{
Arun Prasad, MBBS, DA, FRCA, Paul Tumber, MD, FRCPC, Mario Lupu, MD \\ Anesthesia, Toronto Western Hospital, University of Toronto, Toronto, ON, Canada
}

Purpose: To highlight the potential use of ultrasound (US) for spinal anesthesia in patients with scoliosis and previous back surgery.

Clinical Features: Informed consent has been obtained from the patient. Patients with previous back surgery and scoliosis can be challenging for performing central neuraxial blocks(CNB) (1). Technical difficulty and abandonment of the procedure has been reported in these kind of patients (2). Case Report: A 56 year old female patient was admitted for a total knee arthroplasty under spinal anesthetic. Her medical history included hypothyroidism and obesity with a BMI of 32. She had multiple back surgeries for chronic back pain that included an L5-S1 discectomy, an L4-L5 decompression and laminoplasty with a further decompression for epidural hematoma 2 weeks following the surgery and a further revision of L4-L5 decompression and fusion with cage. Spine X ray is shown in Figure 1. The patient was placed in the sitting position and her back was scanned using a Philips HD 11 ultrasound machine with a 2-5 MHz curved probe. We first identified the sacrum in the longitudinal axis at left paramedian location. The probe was moved cephalad scanning the paramedian views of the spine to identify the laminae and transverse processes. We looked for the best window that would allow us to reach the spinal space with minimal interference to neural tissue or surgical intervention. We avoided the level of spinal instrumentation i.e. L4-5. On the left paramedian approach, the best window at the lowest level was available at L2-L3. Using a standard $25 \mathrm{G}$ Whitacre needle through an introducer, an uneventful spinal was performed in a single attempt at the marked spot for the L2-L3 left paramedian approach. In our practice using US for such patients has improved the odds of success, and has decreased the number of attempts required for a successful spinal. However we realize these are anecdotal findings at this stage and will need large scale randomized studies to substantiate it.

Conclusion: Thus, bedside use of ultrasound in patients with scoliosis and or previous back surgery may aid in performing spinal anesthetic.

References: 1. Can J Anesth 2006; 53: 325-326 2. Der Anaesthesist 1996; 45: 66-9 


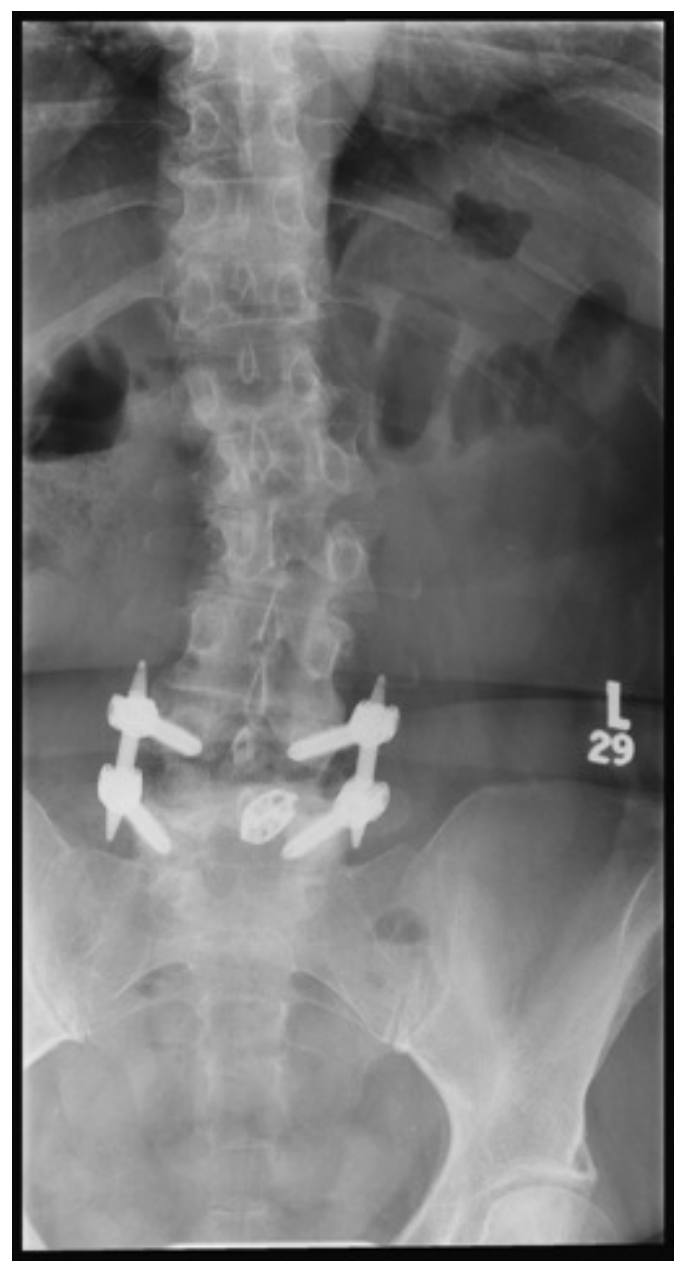

Figure 1: Note the scoliosis with convexity to left and presence of lumbar instrumentation. 